\title{
204.
}

\section{ON A PROBLEM IN THE PARTITION OF NUMBERS.}

[From the Philosophical Magazine, vol. xIII. (1857), pp. 245-248.]

IT is required to find the number of partitions into a given number of parts, such that the first part is unity, and that no part is greater than twice the preceding part.

Commencing to form the partitions in question, these are

$$
1\left|\begin{array}{ll|llllll}
1 & 1 & 1 & 1 & 1 & 1 & 1 & 1 \\
1 & 2 & 1 & 1 & 2 & 2 & 2 & 2 \\
1 & 2 & 1 & 2 & 3 & 4
\end{array}\right| \text { \&c. }
$$

and if we were to proceed to the 4-partitions, each 3-partition ending in 1 would give rise to two such partitions; each 3-partition ending in 2 to four such partitions; each 3-partition ending in 3 to six partitions; and each 3-partition ending in 4 to eight such partitions. We form in this manner the Table:

\begin{tabular}{|c|c|c|c|c|c|c|c|c|c|c|c|c|c|c|c|c|c|}
\hline Number of & 1 & 2 & 3 & 4 & 5 . & 6 & 7 & $\begin{array}{l}\text { en } \\
8\end{array}$ & 9 & $\begin{array}{l}\text { in } \\
10\end{array}$ & 11 & 12 & 13 & 14 & 15 & 16 & Totals \\
\hline 1-partitions & 1 & & & & & & & & & & & & & & & & 1 \\
\hline 2-partitions & 1 & 1 & & & & & & & & & & & & & & & 2 \\
\hline 3-partitions & 2 & 2 & 1 & 1 & & & & & & & & & & & & & 6 \\
\hline -partitions & 6 & 6 & 4 & 4 & 2 & 2 & $\dot{1}$ & 1 & & & & & & & & & 26 \\
\hline -partitions & 26 & 26 & 20 & 20 & 14 & 14 & 10 & 10 & 6 & 6 & 4 & 4 & 2 & 2 & 1 & 1 & 166 \\
\hline
\end{tabular}
\&c. ; 
and we are thus led to the series

$$
\begin{aligned}
& 1, \\
& 1,2, \\
& 1,2,4,6, \\
& 1,2,4,6,10,14,20,26, \\
& \text { \&c. }
\end{aligned}
$$

where, considering 0 as the first term of each series, the first differences of any series are the terms twice repeated of the next preceding series: thus the differences of the fourth series are $1,1,2,2,4,4,6,6$. It is moreover clear that the first half of each series is precisely the series which immediately precedes it; we need, in fact, only consider a single infinite series, $1,2,4,6$, \&c. It is to be remarked, moreover, that in the column of totals, the total of any line is precisely the first number in the next succeeding line.

Consider in general a series $A, B, C, D, E$, \&c., and a series $A^{\prime}, B^{\prime}, C^{\prime}, D^{\prime}, E^{\prime}$, \&c. derived from it as follows:

$$
\begin{aligned}
& A^{\prime}=1 A, \\
& B^{\prime}=2 A, \\
& C^{\prime}=2 A+B, \\
& D^{\prime}=2 A+2 B, \\
& E^{\prime}=2 A+2 B+C, \\
& F^{\prime}=2 A+2 B+2 C, \\
& \& c . ;
\end{aligned}
$$

viz. the first differences of the series $0, A^{\prime}, B^{\prime}, C^{\prime}, D^{\prime}, E^{\prime}$, \&c. are $A, A, B, B, C, C$, \&c. Then multiplying by $1, x, x^{2}$, \&c. and adding, we have

$$
\begin{aligned}
A^{\prime}+B^{\prime} x+C^{\prime} x^{2}+\& c . & =\left(1+2 x+2 x^{2}+\ldots\right)\left(A+B x^{2}+C x^{4}+\& c .\right) \\
& =\frac{1+x}{1-x}\left(A+B x^{2}+C x^{4}+\& c .\right) ;
\end{aligned}
$$

and if we form in a similar manner $A^{\prime \prime}, B^{\prime \prime}, C^{\prime \prime}, D^{\prime \prime}$, \&c. from $A^{\prime}, B^{\prime}, C^{\prime}, D^{\prime}$, \&c. and so on, we have

$$
\begin{aligned}
A^{\prime \prime}+B^{\prime \prime} x+C^{\prime \prime} x^{2}+\& \mathrm{c} . & =\frac{1+x}{1-x}\left(A^{\prime}+B^{\prime} x^{2}+C^{\prime} x^{4}+\& c .\right) \\
& =\frac{1+x}{1-x} \frac{1+x^{2}}{1-x^{2}}\left(A+B x^{4}+C x^{8}+\& c .\right)
\end{aligned}
$$

and so on. Write $A=1$, and suppose that the process is repeated an indefinit number of times, we have

$$
1+\mathfrak{B} x+\left(\mathfrak{c} x^{2}+\mathfrak{D} x^{3}+\& c_{.}=\frac{1+x \cdot 1+x^{2} \cdot 1+x^{4} \cdot \& c .}{1-x \cdot 1-x^{2} \cdot 1-x^{4} \cdot \& c .}\right.
$$


and the coefficients $1, \mathfrak{B}, \mathfrak{C}, \mathfrak{D}, \&$. are precisely those of the infinite series $1,2,4,6$, \&c. We have more simply

$$
1+\mathfrak{B} x+\left(\mathfrak{C} x^{2}+\mathfrak{D} x^{3}+\& c .=\frac{1}{(1-x)^{2}\left(1-x^{2}\right)\left(1-x^{4}\right)\left(1-x^{8}\right) \& c .},\right.
$$

which gives rise to the following very simple algorithm for the calculation of the coefficients :

$1, \quad 2, \quad 3,4, \quad 5, \quad 6, \quad 7, \quad 8, \quad 9, \quad 10,11,12, \quad 13, \quad 14, \quad 15,16$

$0, \quad 0 ; \quad 1, \quad 2, \quad 4, \quad 6, \quad 9, \quad 12, \quad 16, \quad 20, \quad 25,30, \quad 36, \quad 42, \quad 49, \quad 56$

$1, \quad 2, \quad 4, \quad 6, \quad 9, \quad 12, \quad 16, \quad 20, \quad 25, \quad 30, \quad 36,42, \quad 49, \quad 56, \quad 64, \quad 72$

$0, \quad 0, \quad 0,0 ; \quad 1, \quad 2, \quad 4, \quad 6, \quad 10,14,20,26,35, \quad 44, \quad 56, \quad 68$

$1, \quad 2,4,6, \quad 10,14,20,26,35,44,56,68,84, \quad 100,120,140$

$0, \quad 0, \quad 0, \quad 0, \quad 0, \quad 0, \quad 0, \quad 0 ; \quad 1, \quad 2, \quad 4, \quad 6, \quad 10, \quad 14, \quad 20, \quad 26$

$1|2| 4,6|10, \quad 14, \quad 20,26| 36,46, \quad 60, \quad 74, \quad 94, \quad 114, \quad 140, \quad 166 \mid$

\&c.

The last line is marked off into periods of (reckoning from the beginning) 1, 2, 4, 8, \&c.; and by what has preceded, the series which gives the number of 1-partitions, 2-partitions, 3-partitions, \&c. is found by summing to the end of each period and doubling the results; we thus, in fact, obtain (1), 2, 6, 26, 166, 1626, \&c.: and the same series is also given by means of the last terms of the several periods.

The preceding expression for $1+\mathfrak{B} x+\left(\mathfrak{\complement} x^{2}+\& c\right.$. shows that $\mathfrak{B}$, $\mathfrak{\complement}$, \&c. are the number of partitions of $1,2,3,4,5,6$, \&c. respectively into the parts 1,1 , $2,4,8$, \&c.: and we are thus led to-

Theorem. The number of $x$-partitions (first part unity, no part greater than twice the preceding one) is equal to the number of partitions of $2^{x-1}-1$ into the parts $1,1^{\prime}, 2,4, \ldots 2^{x-2}$. Or, again, it is equal to twice the sum of the number of partitions of $0,1,2, \ldots 2^{x-2}-1$ respectively into the parts $1,1^{\prime}, 2,4, \ldots 2^{x-3}$ (where the number of partitions of 0 counts for 1 ).

For example, the partitions of $0,1,2,3$, \&c. with the parts $1,1,2, \ldots$ are

$$
\begin{aligned}
& \text { (.) } \\
& 1,1^{\prime} \\
& 1+1,1+1^{\prime}, 1^{\prime}+1^{\prime}, 2, \\
& 1+1+1,1+1+1^{\prime}, 1+1^{\prime}+1^{\prime}, 1^{\prime}+1^{\prime}+1^{\prime}, 2+1,2+1^{\prime},
\end{aligned}
$$

the numbers of which are $1,2,4,6$. Hence, by the first part of the theorem, the number of 3-partitions is 6 , and by the second part of the theorem, the number of 4-partitions is

$$
2(1+2+4+6),=26 .
$$

2, Stone Buildings, Murch 17, 1857. 